\title{
Natural product provides alternative transport for iron
}

Deficiencies in passive ion-transport proteins give rise to a range of diseases, including anaemias, cystic fibrosis and cardiac arrhythmias. A recent paper in Science has demonstrated that a small-molecule natural product, hinokitiol, transports iron across cell membranes and restores iron handling in several animal models of iron transporter deficiency.

In mammals, systemic iron levels are primarily controlled through regulation of gut absorption, and iron homeostasis is maintained through a network of active and passive transporters expressed on the plasma membrane and subcellular compartments. In the current study, Grillo et al. reasoned that iron concentration gradients created by active transporters would
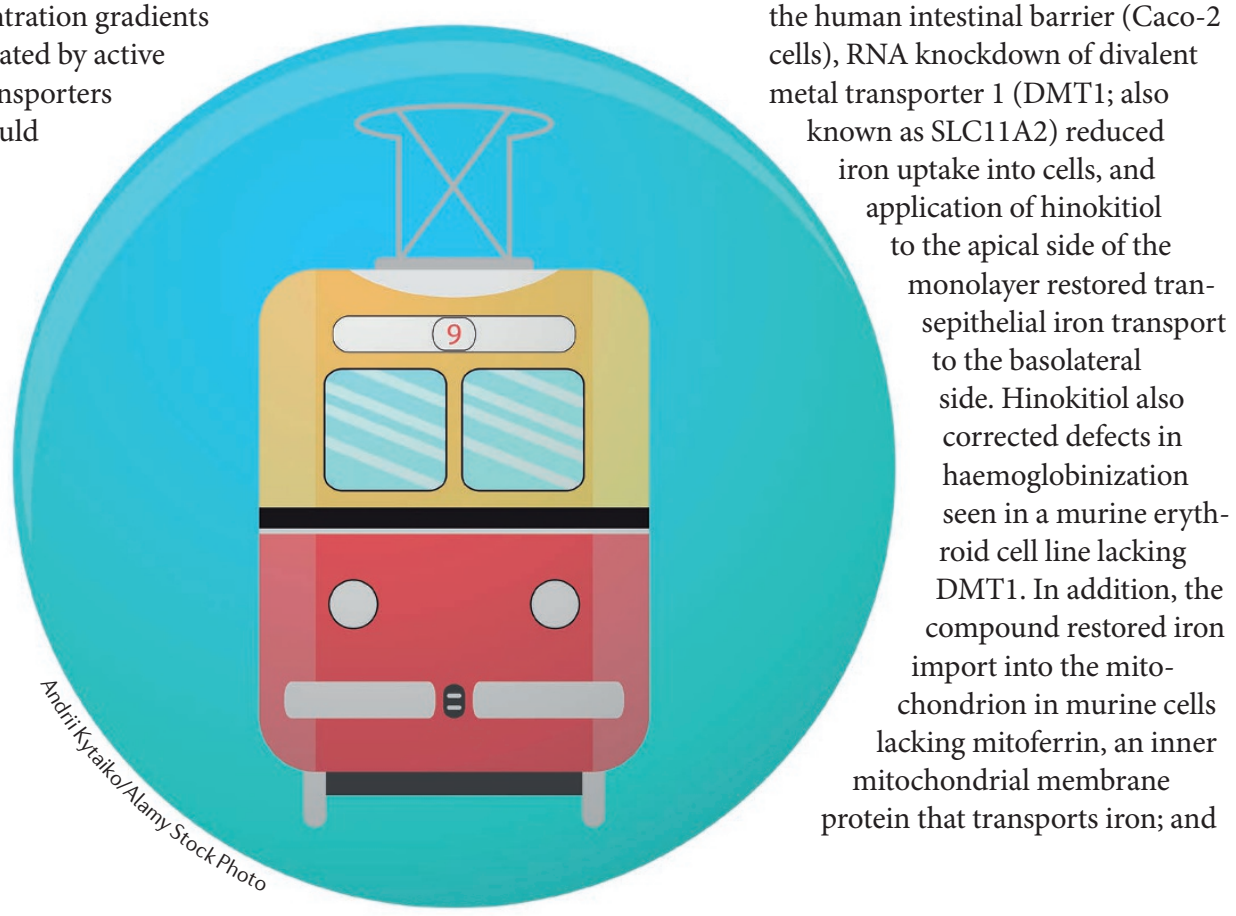

persist in the face of deficiencies in passive transporters and might be harnessed by small molecules that can autonomously mediate ion transport.

Using a screen in yeast, the a lipophilic transmembrane iron transporter. This molecule was originally isolated from the essential oil of the Taiwan Hinoki (Chamaecyparis taiwanensis) tree in 1936 and was known to be a potent chelator of iron.

The authors tested the effect of hinokitiol on mammalian cell lines lacking specific iron transporters to probe iron flux into, within and out of the cell.

In a cell line-derived model of the human intestinal barrier (Caco-2 cells), RNA knockdown of divalent metal transporter 1 (DMT1; also known as SLC11A2) reduced on uptake into cells, and pplication of hinokitiol the apical side of the monolayer restored transepithelial iron transport the basolateral side. Hinokitiol also corrected defects in haemoglobinization seen in a murine erythroid cell line lacking DMT1. In addition, the compound restored iron import into the mitochondrion in murine cells lacking mitoferrin, an inner membrane protein that transports iron; and authors identified hinokitiol as corrected iron efflux from Caco-2 cell monolayers lacking the iron efflux protein ferroportin (FPN1; also known as SLC40A1).

Fluorescent staining experiments indicated that the profile of hinokitiol-mediated iron movement depended upon the site and direction of iron concentration gradients created by endogenous iron transport systems. As such, the authors propose that hinokitiol could work together with physiological iron-handling networks to promote iron homeostasis.

Importantly, administration of a single dose of hinokitiol by oral gavage to Slc11a2-deficient rats and Slc40a1deficient mice significantly improved iron absorption in the gut. Moreover, incubation of Dmt1-deficient zebrafish embryos with the compound promoted haemoglobinization.

Hinokitiol seems to have a good safety profile, as administration of higher doses than those tested in the above study for 2 years had no adverse effect on rats.

This study by Grillo et al. highlights the therapeutic potential of a small-molecule approach that harnesses existing homeostatic networks and could be expanded to address a range of ion transporter deficiencies.

\section{Katie Kingwell}

ORIGINAL ARTICLE Grillo, A. S. et al. Restored iron transport by a small molecule promotes absorption and hemoglobinization in animals. Science 356, 608-616 (2017) FURTHER READING Crielaard, B. J. et al. Targeting iron metabolism in drug discovery and delivery. Nat. Rev. Drug Discov. 16, 400-423 (2017) 